\title{
Remote monitoring system based on C/S and B/S mixed mode
}

\author{
Kaibing Song ${ }^{1, a}$, Yinsong Wang ${ }^{2, b}$ and Dandan Shang ${ }^{3, c}$ \\ ${ }^{1}$ College of Control and Computer Engineering, North China Electric Power University, Baoding, \\ China \\ ${ }^{2}$ College of Control and Computer Engineering, North China Electric Power University, Baoding, \\ China \\ ${ }^{3}$ College of Control and Computer Engineering, North China Electric Power University, Baoding, \\ China \\ a657854440@qq.com, ㅁwys@ncepu.edu.cn, ${ }^{\text {c1 }} 1178956841 @ q q . c o m$
}

Keywords: C/S; B/S; remote monitoring; WINCC; hydraulic turbine governor

\begin{abstract}
With the rapid development of Internet technology, computer and communication technology and control technology, it provides a favorable technical condition for the remote monitoring of control system. In this paper, the working principle and structural characteristics of $\mathrm{C} / \mathrm{S}$ mode and $\mathrm{B} / \mathrm{S}$ mode were analyzed. A design scheme of remote monitoring system based on the mixed mode of $\mathrm{C} / \mathrm{S}$ and $\mathrm{B} / \mathrm{S}$ is presented. To explain this research, the small hydraulic turbine governor system is acted as an example. Running results show that the system has the advantages of simple operation, stable operation, with good real-time and security, and it has certain popularization and application value in engineering.
\end{abstract}

\section{Introduction}

In recent years, with the rapid development of Internet technology, computer and communication technology and control technology, to provide a favorable technical condition for the remote monitoring of control system. The remote monitoring of control system is to use computer to realize the monitor and control of remote production process control system via the network [1]. The computer system which can realize remote monitoring is called remote monitoring system, which is the product of combination of control technology and information network. Remote monitoring system through intranet/Internet remotely monitor and control the production process control system parameters and operating status, elimination the inconvenience due to geographical restrictions, enabling the operator to away from environmental conditions bad, remote and inaccessible etc. working environment, to realize the production process control system of unattended, save a lot of manpower and material resources, and further improve the production process information integration, improve the level of management and decision-making ability. Therefore, the remote monitoring system has been widely studied and applied in industry, agriculture, national defense and other fields [2,3].

With the advance of science and technology, throughout the course of development of remote monitoring system, its structure mainly three kinds of modes, namely host concentrated mode, the client/server $(\mathrm{C} / \mathrm{S})$ mode and the browser/server $(\mathrm{B} / \mathrm{S})$ mode. In the host concentrated mode, the host is typically a powerful computer, remote terminal users share the host CPU resources and database storage of data, data management and transaction processing is highly concentrated [4]. higher initial cost of the System, and the client does not have the processing function, only applies to the scale centralized application, the system scalability is poor, therefore, has gradually been eliminated.

Remote monitoring system based on C/S mode and B/S mode is based on computer network for remote monitoring system of communication platform, with HTTP and TCP/IP technology as the foundation, has the characteristics of simple and effective, has become the two mainstream technology of today's remote monitoring system [5,6]. Domestic and foreign researchers on the remote monitoring system based on $\mathrm{C} / \mathrm{S}$ mode and $\mathrm{B} / \mathrm{S}$ mode, more and more research, and 
achieved good results, but these achievements are mostly used in a single C/S mode or B/S mode, while the use of C/S and B/S mixed mode less. Therefore, on the basis of the working principle and structural characteristics of $\mathrm{C} / \mathrm{S}$ mode and $\mathrm{B} / \mathrm{S}$ mode were analyzed, a design scheme of remote monitoring system based on the mixed mode of $\mathrm{C} / \mathrm{S}$ and $\mathrm{B} / \mathrm{S}$ is presented. To explain this research, the small hydraulic turbine governor system is acted as an example. Running results show that the system has the advantages of simple operation, stable operation, with good real-time and security, and it has certain popularization and application value in engineering.

\section{Structural Analysis Of Remote Monitoring System}

Today's remote monitoring system major can be divided into client/server mode and browser/ server mode according to the structure. Now the working principle and structural characteristics are analyzed respectively.

Client/Server Mode. The client/server mode is mainly composed of two layers of the front-end client and the back-end server, and its system structure is shown in Figure 1.

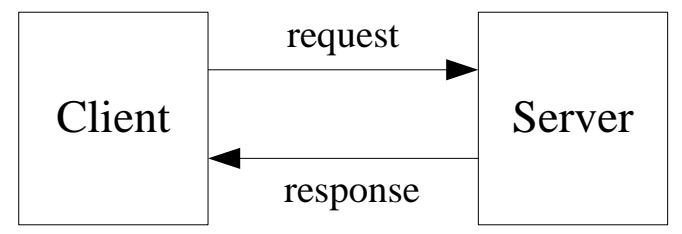

Fig.1 C/S mode system structure

The main function of the client is to interact with the user, provide the graphic interface to the user and communicate with the server. The server mainly provides the statistical inquiry service of the database, the large-scale computation and so on, has the function of data collection, control and communication with the client.

Client/server mode is based on the two tier structure, so the system response time is relatively short, good real-time, to better ensure the consistency of the data [7]. It is suitable for the local area network of the remote monitoring; the system is relatively closed, high security. Dedicated client software has the advantage of a good interactive user interface, and data processing capability is relatively strong. But just because the client need to install special client software, the performance requirements of its clients is relatively high, Special client software is developed for a specific system; the system scalability and maintainability are relatively poor. In the client user more environment may cause the user to wait for a long time, the system will show information blockage, slow database response and other issues.

Browser/Server Mode. Browser/server mode is accompanied by the development of Internet technology and the improvement of C/S mode [8]. It is a three-tier distributed architecture that adds the Web server in the middle of the C/S mode, its system structure as shown in figure 2.

Browser/server mode mainly includes browser, Web server and database server. Browser is to provide a user interface, make service requests to the Web server, and the request content is displayed on the browser. Web server mainly accepts browser service request, to connect to the database server, the data processing request made to the database server, and transfers the data processing result to the browser. The database server accepts the request of the Web server to the data processing realizes the data operation of the database and transmits the result to the Web server.

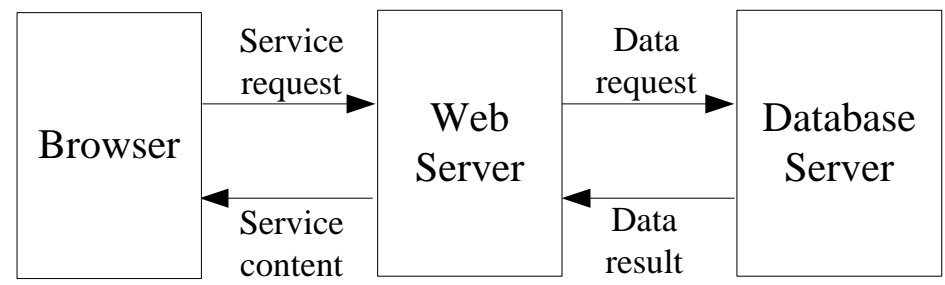

Fig.2 B/S mode system structure 
Browser/server mode is suitable for wide area network and internet remote monitoring [9]. It implements the platform-independent, the user can access the system across different networks and computer platform, has good openness. It is a thin client mode, the client only need to install a Web browser, reducing the burden on the client. Data query, processing and presentation are completed by the server, the upgrade and maintenance of the system is also carried out at the server, and effectively reduces the operation and maintenance work of the system. But the client only installed the Web browser, so the user interface is poor interactivity. While its open characteristics make the system vulnerable to attack, relatively poor security.

\section{Design of Remote Monitoring System}

The purpose of the design of the small hydraulic turbine governor system remote monitoring system is to realize the remote monitoring of the small hydraulic turbine governor system. This requires that the real-time monitoring data of the small hydraulic turbine governor system is transmitted to the local monitoring center by local area network, and it is also required to be transmitted to the remote control center via Internet. Through the data analysis, the operator can judge the real-time operational status and the trend of the small hydraulic turbine governor system, and find the potential problems and timely treatment. According to the above requirements, this paper presents a design scheme of remote monitoring system based on $\mathrm{C} / \mathrm{S}$ and $\mathrm{B} / \mathrm{S}$ mixed mode. In the remote monitoring system design of the mixed mode, you can either bring into play the C/S mode strong processing data capability, good interactive user interface and other advantages, but also can make full use of the cross-platform and good openness of the B/S mode, realize the complementary advantages, foster strengths and circumvent weaknesses, to realize remote monitoring of different regions.

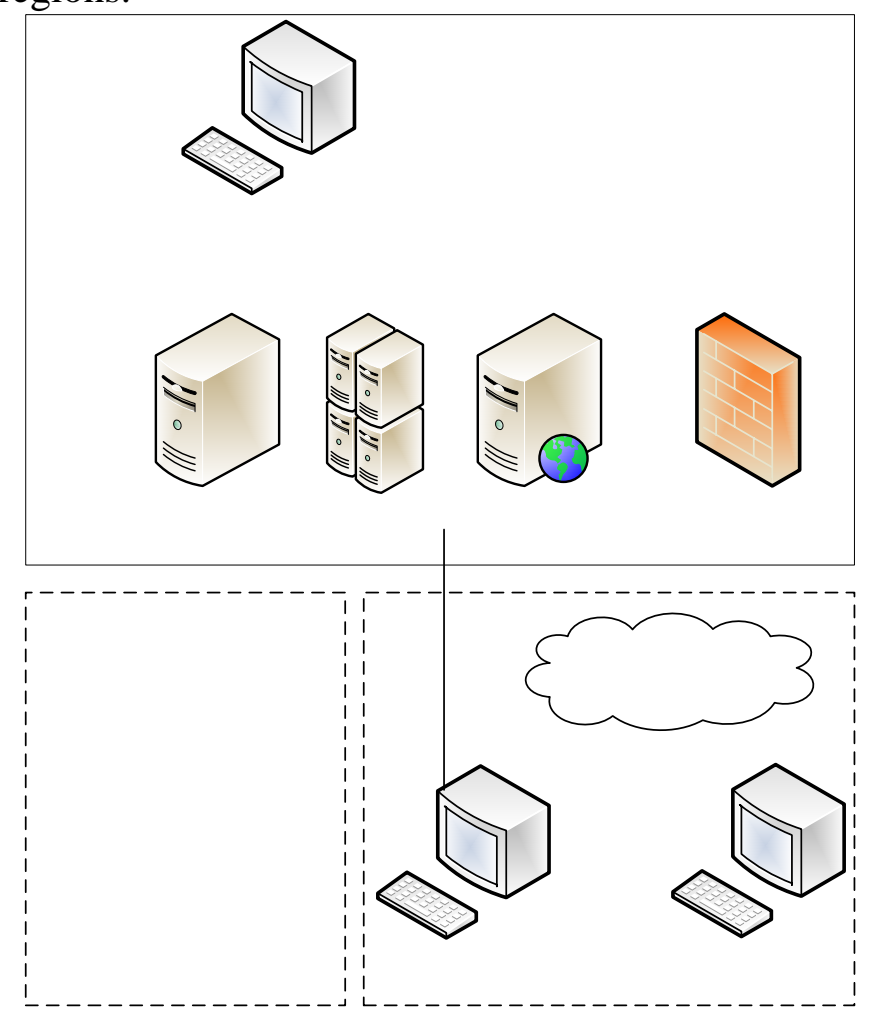

Fig.-3 System architecture

System General Architecture. The overall structure of the remote monitoring system of the small hydraulic turbine governor system is shown in Figure 3, which is composed of three parts: small hydraulic turbine governor system, local monitoring system and remote monitoring system.

Small hydraulic turbine governor system uses OPC (OLE for Process Control) technology to transmit the collected real-time data to the monitoring system [10,11]. Local monitoring system for the local operators to provide monitoring screen, store running state parameters and alarm information. Remote monitoring system provides a monitor screen and running status information 
for the remote operators, to achieve remote control.

Small Hydraulic Turbine Governor System. The control part of the small hydraulic turbine governor system adopts the S7-200 programmable logic controller (PLC) and the corresponding input and output modules of the German SIEMENS company [12]. S7-200 PLC through the I/O module and integrated measurement device to collect the various operating parameters of system, and according to the data collected for data processing, output corresponding control command [13]. Ethernet communication module CP243-1 through network cable to transfer the various operating parameters of system to the monitoring system, and the control information of monitoring system is transmitted to the PLC via the Ethernet communication module; after comprehensive operation, PLC outputs control command, and ultimately realizes the remote monitoring of small hydraulic turbine governor system.

Local Monitoring System. The configuration software SIMATIC WINCC is installed on the computer of the local monitoring system, provides human machine interface for local operators to monitor the operation of small hydraulic turbine governor system, through dynamic graphical image displays system running parameters and trend curves, sets the system's alarm condition, configures alarm messages reports and system operation reports and so on [14]. SIMATIC WINCC through communication configuration software SIMATIC NET exchanges data with the small hydraulic turbine governor system, collects system running state parameters, sends control command and so on [15]. Server SQL 2005 as the background database of the system, used to store the running state real-time data and alarm information, etc., to provide the operator with detailed operating parameters and historical data.

Remote Monitoring System. In this paper, the design of remote monitoring system based on C/S and $\mathrm{B} / \mathrm{S}$ mixed mode can choose the $\mathrm{C} / \mathrm{S}$ mode or $\mathrm{B} / \mathrm{S}$ mode according to the location of the client. The client in the local area network use $\mathrm{C} / \mathrm{S}$ mode remote monitoring system. The client location is far away, not in the local area network; choose B/S mode remote monitoring system.

C/S Remote Monitoring System. SIMATIC WINCC through the configuration client/server system extends the local monitoring system into client/server structure, to achieve the remote monitoring within the local area network. It uses DCOM and ActiveX technology to realize the data communication between the client and the server. The client open the WINCC Project Manager WinCCExplorer, find project server and enter the user name and password, they can get pictures of real-time monitoring, and the corresponding monitoring and control operations according to the user's login authorization.

B/S Remote Monitoring System. WinCC Web Navigator extends the local monitoring system into the browser/server structure, to achieve the remote monitoring in the wide area network and Internet. WinCC Web Navigator uses event driven mode as the data transmission mode. The remote client only needs to install the WinCC Web Navigator Client plug-in to view the monitor screen through the standard browser. Entering the IP address of the Web Navigator Server in the standard browser address bar, there will be appeared a login dialog box. After inputting the user name and password, the client will be connected to the running server, to obtain the real-time monitoring screen, and to realize remote monitoring.

System Security Settings. Network security is an important factor that affects the security of remote monitoring system, especially form the operation of users with control permission. System should be able to reliably confirm the identity of the user, in order to effectively ensure the safe operation of the system. The remote monitoring system through the list of users in the WinCC project for legitimate users to set the account and password, and the operation level of permissions, and graphical interface, labels, and command set the security code to prevent the invasion of illegal users or the unauthorized operation of legitimate users. In addition, the system uses the firewall technology, SSL encryption technology and user name and password login technology and other security measures to ensure the security of network communications.

\section{Summary}

In this paper, the working principle and structural characteristics of C/S mode and B/S mode 
were analyzed. A design scheme of remote monitoring system based on the mixed mode of C/S and $\mathrm{B} / \mathrm{S}$ is presented. To explain this research, the small hydraulic turbine governor system is acted as an example. The remote monitoring system is composed of three parts, which are small hydraulic turbine governor system, local monitoring system and remote monitoring system, and each part was analyzed, and finally describes the security problems of the system. Running results show that the system has the advantages of simple operation, stable operation, with good real-time and security, suitable for local area network and wide area network and Internet remote monitoring, has certain popularization and application value in engineering.

\section{References}

[1] Jianxiu Zhang, Shuo Jin, Xi Zhao and Zhenhu Liang: Web Based Remote Monitoring System for Industrial Wastewater Treatment: submitted to Journal of System Simulation. 2012, 05:1104-1108

[2] Xueya Bao, Jinkuan Wang, Cuijuan An: Remote control system based on internet: submitted to Chinese Journal of Scientific Instrument. 2006,S1:586-588.

[3] Yongguang Ma, Zhenguo Lu, Yongjun Lin: An Internet Based Remote Real-Time Monitoring and Control System for Power Plant: submitted to Power System Technology. 2007,02: 80-83.

[4] Zhen Wang, Changqing Liu, Weipeng An: Research of Mine Monitoring and Control System Based on Hybrid Mode of C/S and B/S: submitted to Industry and Mine Automation. 2008, 01:70-72.

[5] Ximin Zhang, Zhonghai Yin, Guoqing Wang: Design and implication of distributed heat-supply supervision and control system based on TCP/IP network: submitted to Chinese Journal of Scientific Instrument. 2007, 28 ( 5 ) :893-898.

[6] Feng Wu, Chengtie Li, Fenghang He, Deying Gu: Study of Remote Monitor System Based on Web Technology: submitted to Chinese Journal of Scientific Instrument. 2005,S2:241-243.

[7] Yan Ma: Remote monitoring technology based on C/S: submitted to Journal of Southwest China Normal University(Natural Science). 2002,04:501-505.

[8] Hong Liu, Jinzhang Ji, Gencheng Yang: Application of Web-Based HMI/SCADA to Multi-line Ship Lock Monitoring and Control System: submitted to Computer Measurement And Control. 2009, 11:2298-2301.

[9] Zhao Qu, Jianlin Zhu, Xu Lai: Design and implementation of an internet based remote monitoring and diagnosis system for hydroelectric generating set: submitted to Power System Technology. 2004,24:5-9.

[10]Geng Liang, Wen Li: Design of remote monitoring and control system for power plants based on distributed OPC, components linkage and Web Service: submitted to Electric Power Automation Equipment. 2011,10:134-138.

[11]Jinxue Xu, Hongwu Hu,Yumei Liu: Scheme design of remote monitoring and controlling system based on OPC technique: submitted to Journal of Shenyang University of Technology. 2007, 05:574-577

[12] Yang Zhang, Chunwei Cai, Mingjian Sun: S7-200 PLC Principle And Application System Design. China Machine Press. 2007.

[13]Xiaohan Xiang, Yaoyao Liu: Siemens S7-200 PLC fully proficient tutorial. Chemical Industry Press. 2013 
[14]Huabo Liu, Xue Wang, Wenxue He, Yunning Zhang: Configuration software WinCC and its application. China Machine Press. 2013.

[15]Jianfang Jiang, Xue Wang, Wenxue He: Siemens WinCC configuration software engineering technology. China Machine Press. 2013 